\title{
CYSTIC LEUKOENCEPHALOPATHY WITHOUT MEGALENCEPHALY
}

\author{
Eliete Chiconelli Faria', Juliana Harumi Arita', Mirella Maccarini Peruchi², \\ Jaime Lin ${ }^{3}$, Marcelo Rodrigues Masruha ${ }^{4}$, Luiz Celso Pereira Vilanova ${ }^{5}$
}

The coming of magnetic resonance imaging (MRI) into clinical practice added advances in understanding the white matter diseases in children'. Previously, white matter and metabolic diseases were found to have a relatively specific pattern that could help in differential diagnosis, and primary defects in many inborn leukoencephalopathies have been elucidated including disorders from lysossomal storage, amino and organic acids, muscle and mitochondrial dysfunction ${ }^{1,2}$. Recent reports have described an apparently non-progressive condition characterized by severe psychomotor delay with variable degrees of tone and reflex abnormalities, normo- or microcephaly, and MRI findings consisting of bilateral anterior temporal cystic lesions with pericystic abnormal myelination and symmetric patchy lesions with increased signal in the frontal and occipital periventricular white matter regions ${ }^{3}$. Few patients with this condition have been described in literature ${ }^{1-4}$. In some aspects, this condition may resembles other leukoencephalopathies such as "megalencephalic leukoencephalopathy with subcortical cysts" and "leukoencephalopathy with vanishing white matter", due to the presence of subcortical cysts and white matter abnormalities, however, the clinical presentation and other neuroimaging features are distinct ${ }^{3}$.

We describe the clinical, laboratory and neuroimaging characteristics of a patient with this rare entity.

\section{CASE}

A one-year-old boy was born to healthy and non-consanguineous parents at 36 weeks of gestation. There was no family history of neurological diseases. Birth weight was $2,2 \mathrm{~kg}\left(25-50^{\text {th }}\right.$ centile), length $45 \mathrm{~cm}\left(25-50^{\text {th }}\right.$ centile) and head circumference $31 \mathrm{~cm}$ (below $5^{\text {th }}$ centile). Apgar indices were 6 and 9 (at one and ten min). Right after birth, he had transient respiratory discomfort and hypomagnesaemia with no further complications and he was discharged at the third day of life. It was already record-

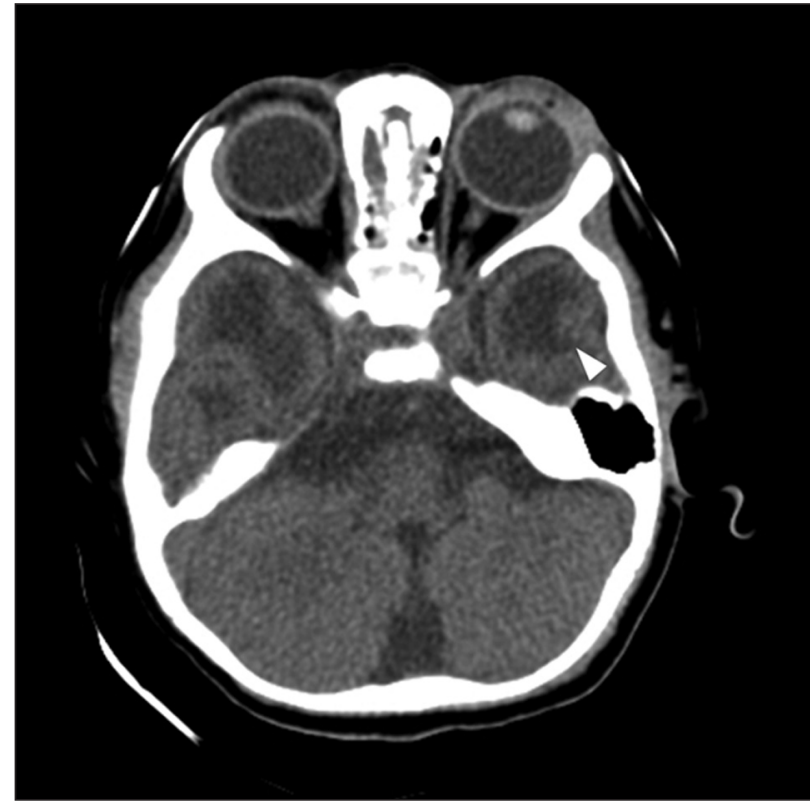

Fig 1. CT image shows well-circumscribed low attenuation lesions in the temporal lobes.

ed that this child was hypotonic, with poor movements and adducted thumbs since the first weeks of life. He also presented a delay in neuropsychomotor acquisitions. The infant was admitted in our service at the age of 12 months. On examination he presented good overall condition with a head circumference of $44 \mathrm{~cm}$ (below $5^{\text {th }}$ centile). Neurological examination revealed a good contact with the examiner but he did not either fix on or catch an offered object. It was also observed increased limb muscle tone, brisk tendon reflexes in contrast with axial and apendicular hypotonia. Evaluation of cranial nerves was normal.

Hematological tests and blood screening for metabolic diseases, including those for concentrations of very long-chain fatty acids, lactate and pyruvate, gave results in the normal ranges. Cerebrospinal fluid lactate concentration was normal. Serolog-

\section{LEUCOENCEFALOPATIA CÍSTICA SEM MEGALENCEFALIA}

Division of Child Neurology / Department of Neurology and Neurosurgery, Federal University of Sao Paulo, Sao Paulo SP, Brazil (UNIFESP-EPM): 'Resident, Department of Pediatrics, UNIFESP-EPM; ${ }^{2}$ Resident, Department of Radiology, Hospital Heliopolis, Sao Paulo SP, Brazil; ${ }^{3}$ Resident, Division of Child Neurology / Department of Neurology and Neurosurgery, UNIFESP-EPM; ${ }^{4}$ Associated Physician, Division of Child Neurology / Department of Neurology and Neurosurgery, UNIFESP-EPM; ${ }^{5}$ Professor and Chairman, Division of Child Neurology / Department of Neurology and Neurosurgery, UNIFESP-EPM.

Received 14 September 2007, received in final form 29 November 2007. Accepted 4 February 2008.

Dra. Eliete Chiconelli Faria - Rua Jacinto de Lima Santos 263 -03738-090 São Paulo SP - Brasil. E-mail: e.faria@ig.com.br 
ical markers for CMV, herpes virus, toxoplasmosis and syphilis were undetectable.

Brainstem auditory evoked potentials detected severe bilateral sensorineural deafness and electroencephalogram showed discrete disorganized background activity with an epileptiform activity in the median line.

Noncontrast-enhanced cranial computed tomography (CT) revealed hipoatenuation of parietal white matter and well-circumscribed temporal subcortical cysts bilaterally (Fig 1). MRI showed bilateral lesions with signal intensity that is isointense to the one of cerebrospinal fluid in both temporal lobes, compatible with cysts (Fig 2) and patchy areas of high signals intensity in the white matter of temporal, frontal and parietal lobes (Fig 3).

Although the patient presented severely handicapped he also continued to make slow developmental progress in motility, speech, and cognitive function.

The hospital ethic commission approved this case report and the parents gave informed consent for publication.

\section{DISCUSSION}

Cystic leukoencephalopathy without megalencephaly was first described by Olivier et al. in 1998 and since then, fewer than 30 cases have been described ${ }^{4}$. The distinctive findings of this rare condition are bilateral temporal lobe cysts combined with a specific pattern of multifocal white matter lesions, normo- or microcephaly, and severe psychomotor retardation in a non-progressive clinical course $e^{2,3}$.

Considerable effort has been done into distinguishing these patients from another leukoencephalopathy known as "megalencephalic leukoencephalopathy with subcortical cysts" (MLC) 33 . Firstly described in 1995, this entity is characterized by dramatic MRI changes including a typical pattern of diffuse white matter lesions and swelling with invariable frontoparietal and anterotemporal subcortical cysts, but mild clinical manifestations initially, development of macrocephaly and functional deterioration later in course ${ }^{2,3,6}$. Mutations in $M L C 1$ gene have been shown to

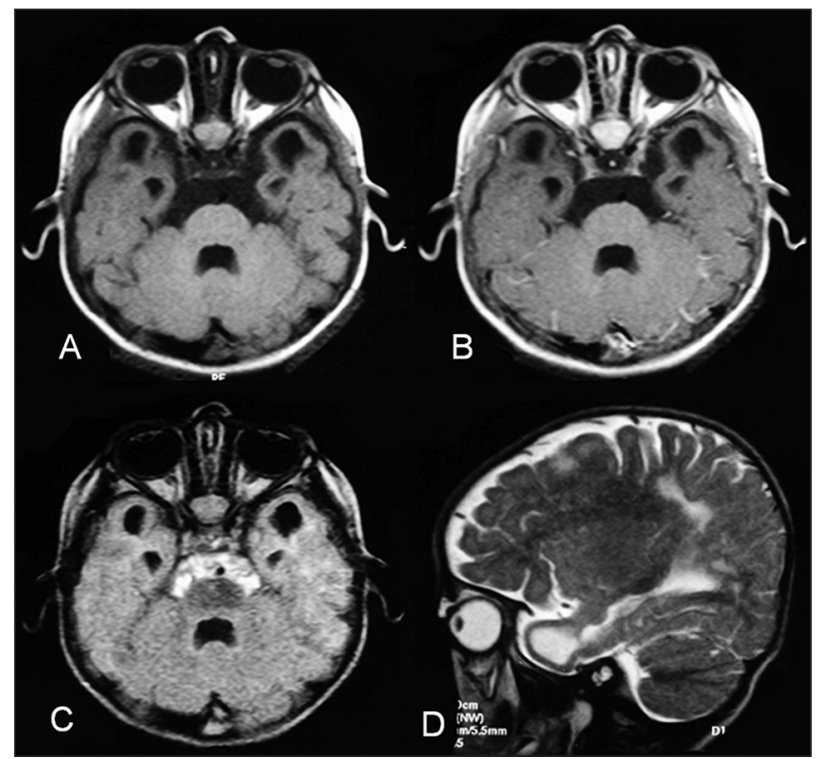

Fig 2. MRI, serial T7-weighted (A), T7-weighted after gadolinium injection (B), fluid-attenuated inversion recovery (FLAIR) sequence (C) axial images and T2-weighted sagital image (D) show bilateral lesions with signal intensity that is isointense to the one of cerebrospinal fluid in both temporal lobes, compatible with cysts.

cause MLC, although there are patients with the condition in whom mutations of MLC 1 cannot be found ${ }^{2-5}$.

In our patient, we did not perform MLC1 testing because of its non-availability; however, clinical and neuroimaging features of cystic leukoencephalopathy without megalencephaly are clearly distinct from MLC. Patients with cystic leukoencephalopathy without megalencephaly are severely involved from the beginning with a non-progressive course, they have normal or small head circumference and MRI findings contrast to the patients with MLC.

Another recently described cystic leukoencephalopathy called "childhood ataxia with central nervous system hypomyelination" (CACH) or "leukoencephalopathy with vanishing white matter" superficially resembles cystic leu-

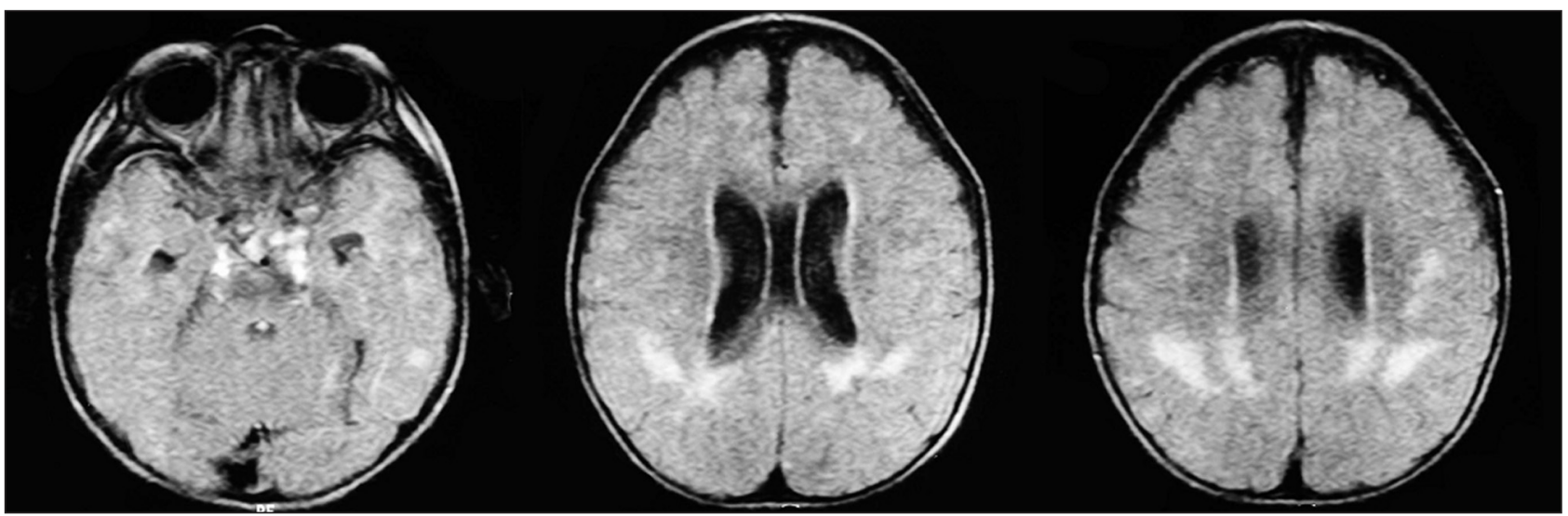

Fig 3. Axial FLAIR sequence from MRI shows patchy areas of high signals intensity in the white matter of temporal, frontal and parietal lobes. 
koencephalopathy without megalencephaly ${ }^{7}$. Patients with $\mathrm{CACH}$ syndrome have extensively dramatic cystic changes in the cerebral white matter; however, the temporal lobes are not preferentially involved ${ }^{7}$. Furthermore, the clinical picture in $\mathrm{CACH}$ syndrome is characterized by a mild initial course, with later deterioration and bouts of sudden decline in function associated with minor head trauma ${ }^{7}$.

It was proposed that the MRI abnormalities found in cystic leukoencephalopathy without megalencephaly could be the result of congenital CMV infection and not a leukodystrophy ${ }^{8}$. Late $\mathrm{CMV}$ infection is one important condition to be considered in the differential diagnosis of cystic leukoencephalopathy without megalencephaly, once it could lead to microcephaly, retarded psychomotor development in a clinically static encephalopathy, and sensorial deafness ${ }^{1,8,9}$. Neonatal signs of intrauterine CMV infection include systemic signs of jaundice, hepatosplenomegaly and petechiae. Most commonly neurological impairment includes sensorineural hearing deficits and decreased vision caused by chorioretinitis. The severity of the neurological impairment may vary from mild learning, behavioral, and motor coordination problems to serious mental deficiency and motor handicap ${ }^{1,9}$. In CMV congenital infection, MRI studies can be normal or demonstrate several abnormal findings such as cerebellar hypoplasia, disorders of neuronal migration and white matter abnormalities including hemorrhage, calcifications and the presence of anterior temporal lobe cysts. Those findings as a result of late infection (third trimester) can be regarded as a result of infection at a time when neuronal migration and cortical plate formation had already occurred resulting in a more selective lesion of glial cells ${ }^{1,910}$.

It is important to note that, in this case, both mother and the patient were tested for $\mathrm{CMV}$ with negative results. Additionally, in our patient neither signs of systemic CMV infection nor signs of calcifications, which are commonly associated with congenital CMV infection, were found. Therefore, according to serological, clinical, and neuroimaging findings, CMV infection is extremely unlikely.

In conclusion, we have reported a patient affected by non-progressive cystic leukoencephalopathy without megalencephaly. The identification of patients with this new white matter disease depends on the recognition of the characteristic of MRI findings of bilateral anterior temporal lobe cystic lesions, with abnormal myelynation in the pericystic region, and patchy symmetric white-matter lesions. It is important to remember that this clinical condition, the neuroimaging confirmation is necessary to avoid mistaken with cerebral palsy.

\section{REFERENCES}

1. Gomes AL, Vieira JP, Saldanha J. Non-progressive leukoencephalopathy with bilateral temporal cysts. Eur J Ped Neurol 2001;5:121-125.

2. Henneke M, Preuss N, Engelbrecht V, et al. Cystic leukoencephalopathy without megalencephaly: a distinct disease entity in 15 children. Neurology 2005;64:1411-1416.

3. Bodensteiner JB, Kerrigan JF, Johnsen SD. Leukoencephalopathy with bilateral anterior temporal lobe cysts. J Child Neurol 2006;21:419-422.

4. Olivier M, Lenard HG, Aksu F, Gärtner J. A new leukoencephalopathy with bilateral anterior temporal lobe cysts. Neuropediatrics 1998;29: 225-228.

5. Grosso S, Cerase A, De Stefano N, et al. Non-progressive leukoencephalopathy with bilateral anterior temporal cysts: a case report and review of the literature. Brain Dev 2005;27:73-77.

6. Van der Knaap M, Barth P, Stroink H. Leukoencephalopathy with swelling and discrepantly mild clinical course in eight children. Ann Neurol 1995;37:324-334.

7. Schiffman R, Moller J, Trapp B. Childhood ataxia with diffuse central nervous system hypomyelination. Ann Neurol 1994;35:331-340.

8. Tatli B, Ozman M, Aydinli N, Caliskan M. Not a new leukodistrophy but a congenital cytomegalovirus infection. J Child Neurol 2005;20:525-527.

9. Van der Knaap M, Vermeulen G, Barkhof F, Hart AA, Loeber JG, Weel JF. Pattern of white matter abnormalities at MR imaging: use of polymerase chain reaction testing of Guthrie cards to link pattern with congenital cytomegalovirus infection. Radiology 2004;230:529-535.

10. Barkovich A, Lindan C. Congenital cytomegalovirus infection of the brain: imaging analysis and embryologic considerations. Am J Neuroradiol 1994;15:703-715. 